\title{
Breathing motion compensated registration of laparoscopic liver ultrasound to CT
}

João Ramalhinho

Maria Robu

Stephen Thompson

Philip Edwards

Crispin Schneider

Kurinchy Gurusamy

David Hawkes

Brian Davidson

Dean Barratt

Matthew J. Clarkson 


\title{
Breathing motion compensated registration of laparoscopic liver ultrasound to $\mathrm{CT}$
}

\author{
João Ramalhinho ${ }^{*} a$, Maria $\operatorname{Robu}^{a}$, Stephen Thompson ${ }^{a}$, Philip Edwards ${ }^{a}$, Crispin Schneider ${ }^{b}$, \\ Kurinchi Gurusamy ${ }^{b}$, David Hawkes ${ }^{a}$, Brian Davidson ${ }^{b}$, Dean Barratt ${ }^{a}$, Matthew J. Clarkson ${ }^{a}$ \\ ${ }^{a}$ Centre for Medical Image Computing, University College London, London WC1E 7JE, UK \\ ${ }^{b}$ Division of Surgery and Interventional Science, UCL Medical School, London WC1E 6BT, UK
}

\begin{abstract}
Laparoscopic Ultrasound (LUS) is regularly used during laparoscopic liver resection to locate critical vascular structures. Many tumours are iso-echoic, and registration to pre-operative CT or MR has been proposed as a method of image guidance. However, factors such as abdominal insufflation, LUS probe compression and breathing motion cause deformation of the liver, making this task far from trivial. Fortunately, within a smaller local region of interest a rigid solution can suffice. Also, the respiratory cycle can be expected to be consistent. Therefore, in this paper we propose a feature-based local rigid registration method to align tracked LUS data with CT while compensating for breathing motion. The method employs the Levenberg-Marquardt Iterative Closest Point (LMICP) algorithm, registers both on liver surface and vessels and requires two LUS datasets, one for registration and another for breathing estimation. Breathing compensation is achieved by fitting a 1D breathing model to the vessel points. We evaluate the algorithm by measuring the Target Registration Error (TRE) of three manually selected landmarks of a single porcine subject. Breathing compensation improves accuracy in $77 \%$ of the measurements. In the best case, TRE values below $3 \mathrm{~mm}$ are obtained. We conclude that our method can potentially correct for breathing motion without gated acquisition of LUS and be integrated in the surgical workflow with an appropriate segmentation.
\end{abstract}

Keywords: Multi-modality fusion, laparoscopic liver ultrasound, computed tomography, image registration, electromagnetic tracking, liver surface digitisation, breathing motion compensation

\section{INTRODUCTION}

In the UK approximately 1800 liver resections are performed annually for primary or meta-static cancer. Liver cancer is a major global health problem and 150,000 patients per year could benefit from liver resection. Currently, approximately $10 \%$ of patients are considered suitable for laparoscopic liver resection, mainly those with small cancers on the periphery of the liver. Potentially, laparoscopic resection has significant benefits in reduced pain and faster recovery for the patient, along with cost savings due to shorter hospital stays. ${ }^{1}$ Larger lesions and those closer to major vascular structures are generally considered high risk for the laparoscopic approach mainly due to the restricted field of view and lack of haptic feedback. Laparoscopic Ultrasound (LUS) has the potential to increasing the safety of this intervention since it is able to image sub-surface structures, such as vessels and tumours. Since several types of tumours are iso-echoic and not visible in LUS, registration of tracked LUS to a pre-operative CT scan has been proposed as a method of image guidance. By having both modalities aligned, critical structures identified in the CT can be overlayed on the laparoscopic view.

Several solutions have been proposed for ultrasound (US) to CT registration of the liver. Methods that register freehand US to CT have been reported, either by correlating US with an US simulation from CT plus the CT signal itself, ${ }^{2}$ or by converting both US and CT to vessel probability maps and correlate them afterwards. ${ }^{3}$ Since these methods use freehand US and assume a large coverage of the liver, they can not be applied in laparoscopy given the restricted probe movement and field of view. As an alternative to freehand US, other authors register 3D US, either by using reconstructed vessels as features ${ }^{4,5}$ or by using segmented liver surface and vessels. ${ }^{6}$ However, 3D US capable probes are currently not available for laparoscopy.

\footnotetext{
*E-mail: joao.ramalhinho.15@ucl.ac.uk
} 


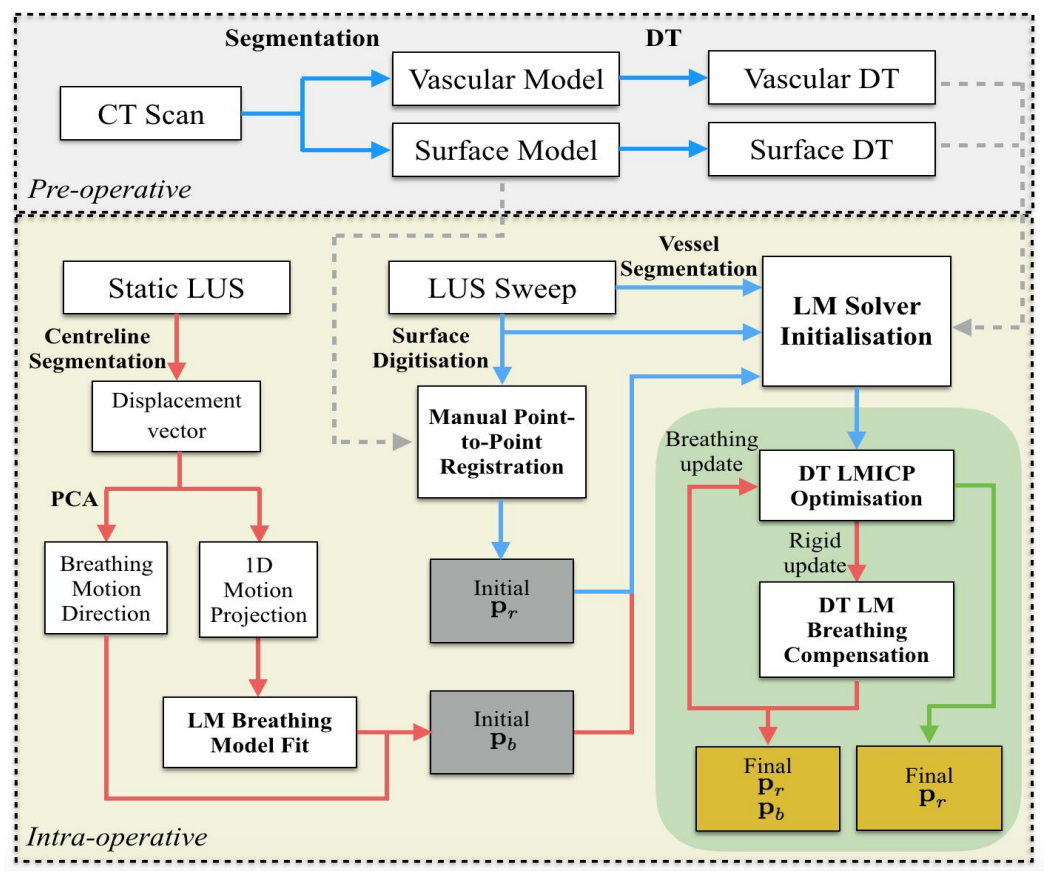

Figure 1. Proposed method workflow. Both rigid and breathing motion compensated approaches are highlighted: green arrows refer to the former; red arrows to the latter; blue arrows represent procedures that are common to both.

Thus far, there have been few studies concerning the specific task of LUS to CT registration. Initial feasibility studies of this concept have been performed with landmark-based registration both on an isolated phantom ${ }^{7}$ and in a full experimental laparoscopic setup. ${ }^{8}$ First results of a surface-based rigid registration on a porcine subject were reported using the Lap-Assistent complete system. ${ }^{9}$ Since in real laparoscopic conditions the liver is subject to deformation due to insufflation and LUS probe compression, the solutions above may not be accurate enough. Results highlighting the possibility of a rigid solution being sufficient locally have been reported on porcine subjects using a vessel-based algorithm. ${ }^{10}$ However, the presented workflow required manual landmark identification, making it difficult to apply in a workable time frame.

During surgery, breathing motion is also a considerable source of deformation that can reach displacements around 12-26mm. ${ }^{11}$ A $4 \mathrm{D}$ model that reconstructs this motion intra-operatively in the laparoscopic environment has been reported. ${ }^{12}$ The method co-registered stationary LUS images to obtain 2D deformation fields and fitted a breathing model based on a correlation metric between consecutive images. Different time varying fields were interpolated along the liver with B-Splines to generate a 3D model along time. Still, the method required a considerable amount of time for both acquisition and reconstruction, which ultimately does not allow for the registration to be updated several times throughout surgery.

We propose a framework for feature-based rigid registration of tracked LUS to CT with compensation for breathing motion. The method registers using one sweep of LUS data, assuming that in a smaller region of interest a rigid solution can be sufficient. ${ }^{10}$ Liver vessels and surface points are used as features. A breathing model is also introduced, since respiratory cycle variation is expected to be consistent throughout surgery and can generate significant displacements of up to $5-25 \mathrm{~mm} .{ }^{12}$ To fit this model a single additional LUS dataset is acquired while holding the probe stationary.

\section{METHODS}

The method proposed in this paper employs the LMICP algorithm ${ }^{13}$ and poses the registration problem as the minimisation of the distance between an US point set and a CT segmented model using its Distance Transform (DT). An overview is shown in Figure 1. 


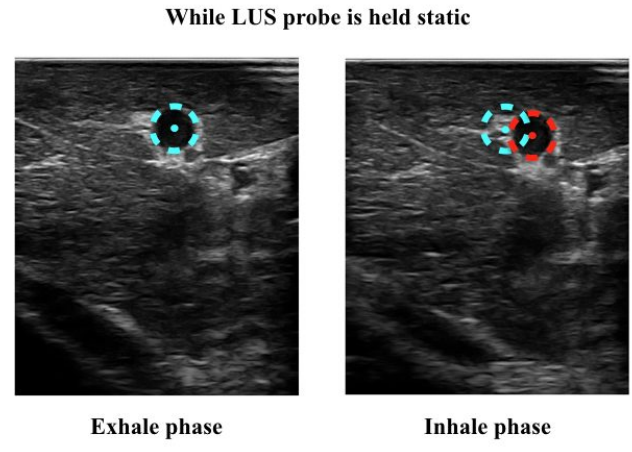

(a)

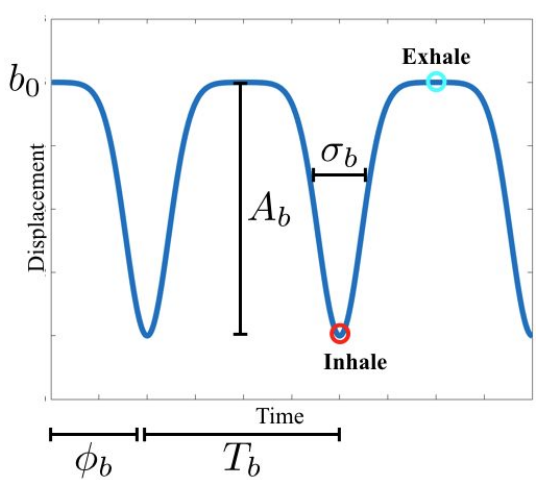

(b)

Figure 2. Breathing motion model: (a) Example LUS images where the displacement of vessels indicates the range of breathing motion from maximum exhale (left) and maximum inhale (right); (b) We propose a simple one-dimensional breathing model that describes the displacement of vessel structures over time.

Pre-operatively, standard triphase clinical CT scans of the liver are segmented and the liver surface and major vessels extracted. Two separate DTs are computed from the liver surface and vessel tree. Intra-operatively, a LUS sweep of data is acquired and both surface and vessel points are extracted. These points form the input to the registration algorithm which comprises a loop of two Levenberg-Marquardt (LM) optimisers, one that solves the distance minimisation problem by finding a rigid transformation defined by parameters $\mathbf{p}_{r}$ and another by compensating breathing motion with parameters $\mathbf{p}_{b}$. The parameter vector $\mathbf{p}_{r}$ is initialised by manually aligning the LUS surface data with the CT surface model using point picking. The parameter vector $\mathbf{p}_{b}$ is initialised while holding the probe stationary and fitting a breathing motion model. These steps are now described in detail.

\subsection{Breathing motion modelling}

We consider breathing motion as a periodic displacement with constant temporal frequency which reaches a maximum at inhale and a minimum at exhale (Figure 2.(b)). We describe this behaviour in equation (1). This model is similar to the one in, ${ }^{14}$ but instead of modelling the inhale-exhale sequence with even powers of a cosine, it regards inhale peaks as narrow gaussian curves and the exhale baseline as the space between them. Five parameters define our models: $A_{b}$, the amplitude of the maximum displacement at inhale; $b_{0}$, the baseline displacement during exhale, $\sigma_{b}$, the width of the gaussian peaks; $T_{b}$, the breathing period that separates each peak center in time; $\phi_{b}$, the breathing cycle phase offset at the origin. The variable $g$ is a constant defined as the number of breathing cycles expected to occur in a time window $\left[t_{1} \ldots t_{n}\right]$ given a period $T_{b}$.

$$
B(t)=b_{0}-\sum_{k=1}^{g} A_{b} \exp \left(\frac{-\left(t-T_{b} k-\phi_{b}\right)^{2}}{2 \sigma_{b}}\right), \quad g=\frac{t_{n}-t_{1}}{T_{b}}
$$

In order to initialise $\mathbf{p}_{b}$, this equation is fitted to the movement in time of a clearly distinguishable feature captured in a time series of LUS images. Given the absence of probe movement, it is expected that breathing will displace the LUS image features with a defined frequency and direction. By applying a Principal Component Analysis (PCA) in a segmented feature such as vessel centreline (Figure 2.(a)) this direction along with its projection over time is estimated. Breathing model parameters are obtained through a LM optimised fit of equation (1) to this principal projection. The principal direction becomes an additional breathing parameter, the breathing motion direction $\theta_{b}$.

\subsection{Ultrasound Image Processing}

From the LUS dataset that is to be registered, liver surface and vessel contours are acquired. The position of the US points in tracker space is given by an electromagnetic (EM) tracker attached to the probe and the US calibration. Surface points are digitised as in ${ }^{15}$ since the probe is in direct contact with the liver during acquisition. Considering a LUS sweep of $n$ images, the surface "corners" of image 1 and image $n\left\{p_{1} \ldots p_{4}\right\}$ are 


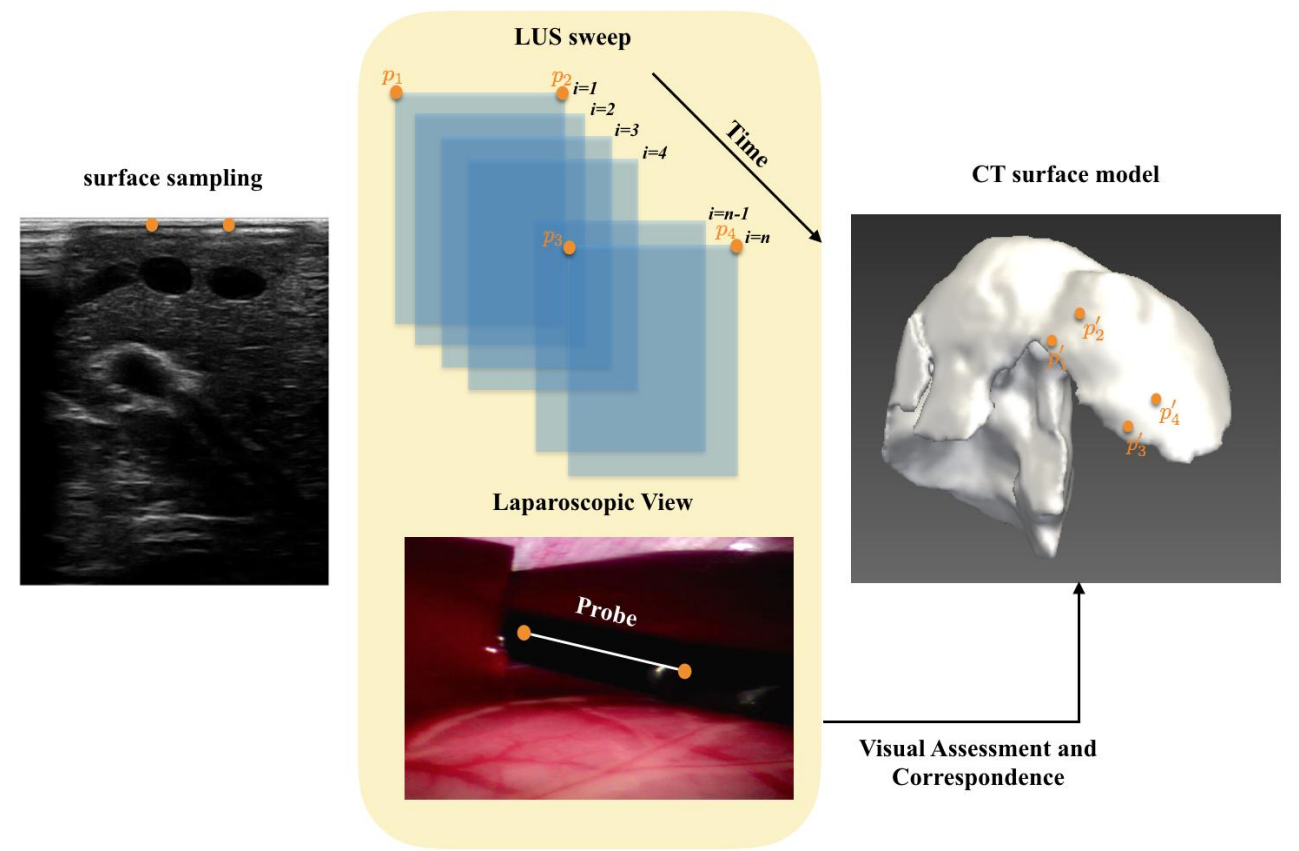

Figure 3. Surface point sampling and application in initial rigid alignment. (Left) orange dots represent surface digitised points from the probe interface in contact with the liver. (Middle) first and last surface point of first and last image of the sweep are matched to the corresponding real probe position through video observation. (Right) points are placed in the CT surface model for further point-to-point registration.

matched to a set $\left\{p_{1}^{\prime} \ldots p_{4}^{\prime}\right\}$ on CT space (See Figure 3.). These CT points are set manually through observation of the probe trajectory in the laparoscopic video, and a point-to-point registration is used to obtain an initial rigid alignment $\left(\mathbf{p}_{r}\right.$ initialisation in Figure 1). Vessel outlines are manually extracted by point picking from each individual LUS image. Other authors have previously published methods on automatic vessel segmentation from ultrasound images. ${ }^{16,17}$

\subsection{Registration}

The error function to minimise in both LM optimisers is given by equation (2), where $D_{f}(\mathbf{x})$ is the interpolated distance of vector $\mathbf{x}$ in the DT of feature $f$ and $T(\mathbf{a} ; \mathbf{x})$ the rigid transformation of $\mathbf{x}$ by parameters $\mathbf{a}$. The distance of the LUS points $\mathbf{x}_{U S}=\left[\mathbf{x}_{v}, \mathbf{x}_{s}\right]$ is computed separately for vessel points $\mathbf{x}_{v}$ and surface points $\mathbf{x}_{s}$ with their respective DT. Two weighting factors $w_{v}$ and $w_{s}$ are used to balance the importance of each feature.

$$
E\left(\mathbf{x}_{U S}\right)=\sum_{i=1}^{n_{s}} w_{s} D_{s}\left(T\left(\mathbf{p}_{r} ; x_{s_{i}}\right)\right)+\sum_{k=1}^{n_{f r a m e s}} \sum_{i=1}^{n_{v k}} w_{v} D_{v}\left(T\left(\mathbf{p}_{r} ; x_{v_{k, i}}-B_{v}\left(t_{k}\right) \cdot \theta_{b}\right)\right)
$$

During optimisation, the US space points $\mathbf{x}_{U S}$ are both rigidly transformed by $\mathbf{p}_{r}$ and compensated with the breathing function $B_{v}(t)$ modelled by $\mathbf{p}_{b}$. The two optimisers are called in a loop which iteratively updates the two parameter vectors until $E\left(\mathbf{x}_{U S}\right)$ converges. $B_{v}(t)$ is a function dependent on time: to each set of $n_{v k}$ points segmented in a LUS image acquired at time $t_{k}$ there is a corresponding correction $B_{v}\left(t_{k}\right)$. To prevent the optimiser from using this correction to compensate surface compression induced by the probe, no compensation is applied to $\mathbf{x}_{s}$.

Although the breathing is defined by six parameters, only four are optimised: the amplitude $A_{b}$, phase offset $\phi_{b}$, and 3D direction $\theta_{b}$ defined by two angles $\alpha_{b}$ and $\beta_{b}$. The two remaining parameters $T_{b}$ and $\sigma_{b}$ are considered non-optimisable since they are expected to remain constant during the procedure. The exhale offset $b_{0}$ is neglected since it is directly correlated with rigid translations. 


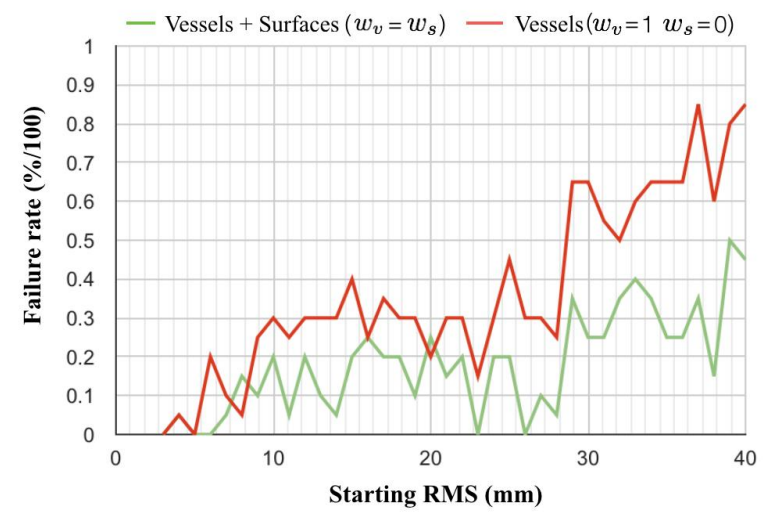

Figure 4. LMICP registration failure rates when using either Surfaces+Vessels or just Vessels, as the initial mis-alignment is increased. We used synthetic LUS data, generated from the CT scan, generated random mis-registrations and the proposed rigid registration method used to re-register the synthetic LUS data to the original CT data.

\subsection{Experiments}

Data from a single porcine subject was used in order to evaluate the registration accuracy of the proposed approach. Surface and vessel models were segmented* from an abdominal triphase CT scan under pneumoperitoneum (insufflated abdomen). LUS images were acquired at a rate of $10 \mathrm{~Hz}$ using an Analogic ${ }^{\dagger}$ SonixMDP and a Vermon ${ }^{\ddagger}$ LP7 linear probe which was tracked at a rate of $40 \mathrm{~Hz}$ by an NDI Aurora ${ }^{\S}$ electromagnetic tracker.

Three experiments were performed. Firstly, an experiment with synthetic data was performed in order to assess the rigid LMICP performance when using vessels and surface data compared to just using vessels. The synthetic dataset was generated by intersecting the CT segmented vascular and surface models with a set of EM tracked planes. Random rigid mis-registrations were induced in the dataset and LMICP was tested with both segmented features $\left(w_{s}=w_{v}\right)$ or just vessels $\left(w_{s}=0 ; w_{v}=1\right)$. The number of times the algorithm failed in recovering the initial configuration was counted after inducing 760 mis-registrations: 20 for each initial RMS interval of $1 \mathrm{~mm}$ within the range [2-40] $\mathrm{mm}$.

The remaining two experiments were applied on the porcine liver data. Seven LUS datasets were acquired, four while holding the probe stationary to measure and fit the breathing model and three sweeping it along the left lobe. Vessel contours were manually segmented from the three sweeps and an initial rigid alignment obtained through the method of section 2.2. In a second experiment, the performance of rigid LMICP was again evaluated using the same feature combinations $\left(w_{s}=w_{v}\right)$ and $\left(w_{s}=0 ; w_{v}=1\right)$ on the three LUS sweeps. For comparison, the accuracy was measured as the tracking error (TRE) of 3 manually selected landmarks (L1, L2, L3) on both the CT models and LUS images.

In the third experiment, LMICP with breathing compensation was tested on the same sweeps using the same feature weight combinations and comparison study. In order to obtain a breathing model estimate, one vessel centreline was manually extracted from each of the four static LUS datasets and the method of section 2.1 applied. The same TRE measurements as in the second experiment were repeated in order to compare breathing compensated and rigid approaches.

\section{RESULTS}

Results of the first experiment are summarised in the Figure 4: registration failure rates are plotted against starting RMS errors of induced mis-registration. Each failure rate value is measured as the percentage of unsuccessful registrations in which the starting RMS error was within a $1 \mathrm{~mm}$ window. As expected, increased

\footnotetext{
*www.visiblepatient.com

${ }^{\dagger}$ www.analogicultrasound.com

${ }^{\ddagger}$ www. vermon.com

$\S_{\text {Www.ndigital.com }}$
} 


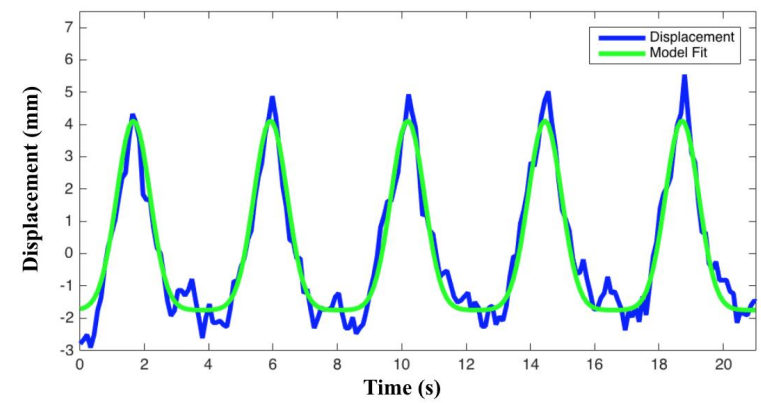

Figure 5. A single example of fitting the breathing motion model to the signal extracted from a moving vessel.

initial RMS error resulted in higher failure rates for both weight combinations. Also, LMICP using both surfaces and vessels produced an overall lower failure rate.

TRE results for the second experiment are listed in the left part of Table 1. The location of the landmarks in the CT segmented models is represented in Figure 6. In all the three sweeps, results for L1 were less accurate than for both L2 and L3. Using LMICP with vessel contours and surface points resulted in a slightly lower mean TRE error compared to using just vessels. In each dataset this TRE improvement was observed in two landmarks out of three.

Two sets of results are presented for the third experiment. Firstly, Table 2. summarises the results of breathing model fitting to each of the four datasets. As expected, among all datasets a very small variance was observed in the parameters $T_{b}$ and $\sigma_{b}$, the ones we considered as non-optimisable. This consistency was also observed in the absolute amplitude values. The principal direction of movement and phase results are different between datasets. Figure 5. shows an example of the breathing model fitted to the projection of the vessel movement.

TRE results of the third experiment for the breathing compensated LMICP are displayed in the right side of Table 1. In terms of mean TRE, results showed in the best case an improvement of $1.5 \mathrm{~mm}$ and in the worst case $0.01 \mathrm{~mm}$. The same trend where the combination of both features yields slightly lower TRE values than just using vessels was again repeated except for sweep 2, where using vessels only achieved the best TRE for L1. Moreover, although the mean TRE was always lower than in the rigid results of the second experiment, in some cases not all landmark TRE were lower than before. When using just vessels, improvements were obtained in $55 \%$ of the measurements versus $77 \%$ when using both features. The highest accuracy value obtained was of $2.7 \mathrm{~mm}$ for L2 in Sweep 3 using equal feature weights. 3D results of this registration solution are shown in Figure 6.

\section{DISCUSSION AND CONCLUSIONS}

Results from the first experiment indicate that using surface data in addition to vessels improves the convergence of the algorithm. This can be physically explained by the fact that a surface patch constrains the rotation space

Table 1. TRE values obtained after running LMICP on data from three LUS sweeps. Results on the left refer to rigid LMICP; results on the right refer to breathing compensated LMICP.

\begin{tabular}{lccccccccc}
\hline & Method & \multicolumn{4}{c}{ Rigid } & \multicolumn{4}{c}{ Breathing Compensated } \\
\hline & TRE $(\mathbf{m m})$ & L1 & L2 & L3 & Mean & L1 & L2 & L3 & Mean \\
\hline \hline \multirow{2}{*}{ Sweep 1 } & Vessels & 12.3 & 6.3 & 7.0 & $\mathbf{8 . 5}$ & 12.3 & 5.5 & 6.8 & $\mathbf{8 . 2}$ \\
& Vessels + Surface & 11.5 & 4.1 & 8.3 & $\mathbf{8 . 0}$ & 8.2 & 3.4 & 7.7 & $\mathbf{6 . 4}$ \\
\hline \multirow{2}{*}{ Sweep 2 } & Vessels & 10.6 & 6.2 & 4.2 & $\mathbf{7 . 0}$ & 5.5 & 5.9 & 4.5 & $\mathbf{5 . 3}$ \\
& Vessels + Surface & 12.9 & 4.3 & 3.1 & $\mathbf{6 . 7}$ & 10.9 & 5.3 & 4.0 & $\mathbf{6 . 7}$ \\
\hline \multirow{2}{*}{ Sweep 3 } & Vessels & 11.0 & 5.6 & 5.2 & $\mathbf{7 . 3}$ & 11.7 & 6.1 & 4.0 & $\mathbf{7 . 3}$ \\
& Vessels + Surface & 13.7 & 4.0 & 3.9 & $\mathbf{7 . 2}$ & 13.1 & 2.7 & 2.8 & $\mathbf{6 . 2}$ \\
\hline
\end{tabular}




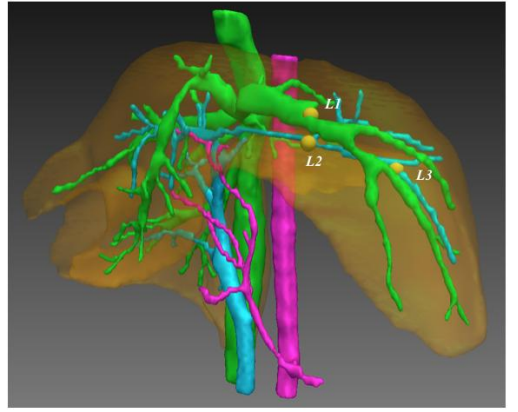

(a)

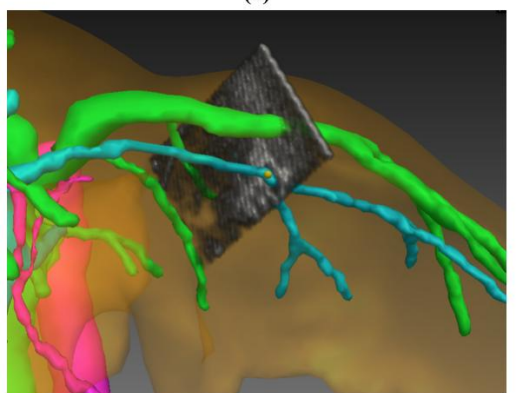

(c)

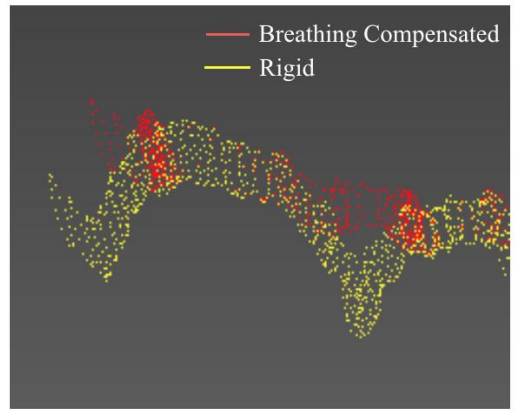

(b)

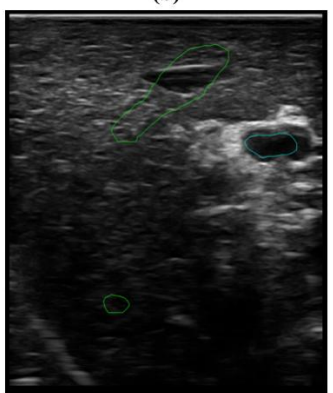

(d)

Figure 6. Registration results of breathing compensated LMICP using vessel and surface features in LUS sweep 3 . (a) The segmented CT model of the subject with manually set landmarks L1, L2 and L3. (b) 3D Breathing compensation results on a subset of the segmented vessel point cloud in tracker space: yellow represents rigid; red represents compensated. (c) A registered LUS image from the sweep aligned with the CT model. (d) the same slice as in (c), but from a 2D perspective.

of the vessels and helps them align with the correct vessel tree, or that the vessel data constrains the sliding of the surface to a more specific solution. Without surfaces, the vessel data extracted from US would frequently lock onto the wrong branch of the vessel tree in CT.

Fitting a breathing model worked well and validates the assumption that breathing motion has a periodic movement with approximately constant frequency and inhale peak width. We proposed gaussian peaks instead of co-sine even powers ${ }^{14}$ to avoid optimising integer parameters during the fitting step. From the remaining parameters, only the amplitude showed similar results between datasets. The variation among phase results could be explained by the fact that the residual difference between each dataset in $T_{b}$ and $\sigma_{b}$ could propagate and generate a considerable time offset. The variance in breathing direction can be explained by three factors: inaccuracy of the segmentation, probe compression and movement during each acquisition. For registration we initialised: $A_{b}$ with $0 ; T_{b}, \sigma_{b}$ and $\theta_{b}$ with the mean of the four datasets; phase $\phi_{b}$ with the value obtained from the dataset acquired closest in time to the three LUS sweeps. Breathing amplitude starts as zero to allow the algorithm to attempt registration without breathing compensation at the start of optimisation. The mean direction is used expecting the optimiser to find a similar direction to the ones measured through PCA. The chosen value for the phase intends to minimise the already mentioned time error propagation induced by the

Table 2. Breathing model fitting results in four static LUS datasets. Offset values $b_{0}$ are not relevant for optimisation and therefore omitted.

\begin{tabular}{lcccccc}
\hline & $\alpha_{b}\left(^{\circ}\right)$ & $\beta_{b}\left(^{\circ}\right)$ & $A_{b}(\mathrm{~mm})$ & $T_{b}(s)$ & $\sigma_{b}$ & $\phi_{b}(s)$ \\
\hline \hline Static 1 & 10.0 & 8.1 & 6.5 & 4.27 & 0.27 & 3.6 \\
Static 2 & -6.6 & 16.9 & 5.2 & 4.27 & 0.27 & 3.5 \\
Static 3 & 6.1 & -22.9 & 5.8 & 4.27 & 0.27 & 1.5 \\
Static 4 & 21.3 & 13.6 & 6.3 & 4.26 & 0.28 & 2.3 \\
\hline
\end{tabular}


small differences in $T_{b}$ and $\sigma_{b}$ between measurements.

TRE values of both the second and third experiment agree with the hypothesis that adding digitised surface data to the registration yields a better accuracy. It is important to note that accurate validation for this method is not a trivial task, as the landmark picking procedure is difficult and therefore error prone. In the specific case of the subject used, some improvement could be made to the CT segmentation, since the generated CT model showed some differences to what could be observed on the LUS. We found a mis-segmentation in the CT model, possibly due to lack of contrast in the original CT data, which made it difficult to identify the precise location of the landmark L1. However, the breathing compensated approach shows some quantitative improvements when compared with just using rigid registration. This idea is corroborated by the visual results in Figure 6.(b): from yellow to red point clouds, there is a clear periodic component attenuation. Also, the compensation was improved by using both vessels and surfaces, suggesting the surfaces produced a regularising effect.

These preliminary results are encouraging given the simplicity and in principle, the ease of integration into the surgical workflow. The method only requires 2 LUS datasets; one stationary sample to estimate the initial breathing model, and another for the registration. In the best cases of L2 and L3, accuracies around 3mm are obtained, which is reasonable for an almost rigid solution in laparoscopic environment, where compression by the LUS probe is still a highly significant non-rigid component. Furthermore, any vessel segmentation algorithm can be coupled to the proposed framework.

Going forward, we intend to apply our method on clinical data and perform a more complete validation. This would provide more insight into how a simple periodic breathing compensation can improve rigid registration based on digitised surfaces and vessels. To improve our breathing model, one possibility is an extension to 2D, similar to Nakamoto et al., ${ }^{12}$ which would enable a more realistic motion compensation. However, in order to avoid large computation times and a complex workflow, the modelling would be confined to the liver region used in registration.

\section{ACKNOWLEDGMENTS}

This publication presents independent research funded by the Health Innovation Challenge Fund (HICF-T4-317), a parallel funding partnership between the Wellcome Trust and the Department of Health. The views expressed in this publication are those of the author(s) and not necessarily those of the Wellcome Trust or the Department of Health. DJH received funding from EPSRC EP/F025750/1. SO and DJH receive funding from EPSRC EP/H046410/1 and the National Institute for Health Research (NIHR) University College London Hospitals Biomedical Research Centre (BRC) High Impact Initiative. We would like to thank NVidia Corporation for the donation of the Quadro K5000 and SDI capture cards used in this research.

\section{REFERENCES}

1. Croome, K. P. and Yamashita, M. H., "Laparoscopic vs open hepatic resection for benign and malignant tumors: An updated meta-analysis," Archives of Surgery 145(11), 1109-1118 (2010).

2. Wein, W., Brunke, S., Khamene, A., Callstrom, M. R., and Navab, N., "Automatic CT-ultrasound registration for diagnostic imaging and image-guided intervention," Medical image analysis 12(5), 577-585 (2008).

3. Penney, G. P., Blackall, J. M., Hamady, M., Sabharwal, T., Adam, A., and Hawkes, D. J., "Registration of freehand 3D ultrasound and magnetic resonance liver images," Medical image analysis 8(1), 81-91 (2004).

4. Aylward, S. R., Jomier, J., Guyon, J.-P., and Weeks, S., "Intra-operative 3D ultrasound augmentation," in [Proceedings, 2002 IEEE International Symposium in biomedical imaging.], 421-424, IEEE (2002).

5. Lange, T., Eulenstein, S., Hünerbein, M., and Schlag, P.-M., "Vessel-based non-rigid registration of MR/CT and 3D ultrasound for navigation in liver surgery," Computer Aided Surgery 8(5), 228-240 (2003).

6. Nam, W. H., Kang, D.-G., Lee, D., Lee, J. Y., and Ra, J. B., "Automatic registration between 3d intraoperative ultrasound and pre-operative ct images of the liver based on robust edge matching," Physics in Medicine and Biology 57(1), 69 (2012). 
7. Bao, P., Warmath, J., Galloway, R., and Herline, A., "Ultrasound-to-computer-tomography registration for image-guided laparoscopic liver surgery," Surgical Endoscopy And Other Interventional Techniques 19(3), 424-429 (2005).

8. Kruecker, J., Viswanathan, A., Borgert, J., Glossop, N., Yang, Y., and Wood, B. J., "An electro-magnetically tracked laparoscopic ultrasound for multi-modality minimally invasive surgery," International Congress Series 1281, 746 - 751 (2005). \{CARS\} 2005: Computer Assisted Radiology and SurgeryProceedings of the 19th International Congress and Exhibition.

9. Martens, V., Besirevic, A., Shahin, O., and Kleemann, M., "Lapassistent: computer assisted laparoscopic liver surgery," in [Proceedings of biomedizinischen technik (BMT) conference], (2010).

10. Song, Y., Totz, J., Thompson, S., Johnsen, S., Barratt, D., Schneider, C., Gurusamy, K., Davidson, B., Ourselin, S., Hawkes, D., and Clarkson, M., "Locally rigid, vessel-based registration for laparoscopic liver surgery," International Journal of Computer Assisted Radiology and Surgery, 1-11 (2015).

11. Rohlfing, T., Maurer Jr, C. R., O'Dell, W. G., and Zhong, J., "Modeling liver motion and deformation during the respiratory cycle using intensity-based free-form registration of gated mr images," in [Medical Imaging 2001], 337-348, International Society for Optics and Photonics (2001).

12. Nakamoto, M., Hirayama, H., Sato, Y., Konishi, K., Kakeji, Y., Hashizume, M., and Tamura, S., "Recovery of respiratory motion and deformation of the liver using laparoscopic freehand 3d ultrasound system," Medical image analysis 11(5), 429-442 (2007).

13. Fitzgibbon, A. W., "Robust registration of 2D and 3D point sets," in [British Machine Vision Conference], 662-670 (2001).

14. Mountney, P. and Yang, G.-Z., "Motion compensated slam for image guided surgery," in [Medical Image Computing and Computer-Assisted Intervention-MICCAI 2010], 496-504, Springer (2010).

15. Miller, K. E., Ondrake, J. E., Pheiffer, T. S., Simpson, A. L., and Miga, M. I., "Utilizing ultrasound as a surface digitization tool in image guided liver surgery," Proc. SPIE 8316, 83163D-83163D-7 (2012).

16. Guerrero, J., Salcudean, S., McEwen, J., Masri, B., and Nicolaou, S., "Real-time vessel segmentation and tracking for ultrasound imaging applications," IEEE Transactions on Medical Imaging 26, 1079-1090 (Aug 2007).

17. Anderegg, S., Peterhans, M., and Weber, S., "Ultrasound segmentation in navigated liver surgery.," in [CURAC], 173-178 (2010). 